REVIEW ARTICLE

\title{
Electrical Cardioversion: A Review
}

\section{Max E Valentinuzzi, EE, $P h D^{1,2^{*}}$ and Luis Aguinaga Arriascu, $M D^{3}$}

${ }^{1}$ Emeritus Professor, Universidad Nacional de Tucumán, Argentina

${ }^{2}$ Emeritus Investigator of CONICET, Argentina

${ }^{3}$ Director of the Arrhythmias Service, Del Parque Clinic San Miguel de Tucumán, Argentina

*Corresponding author: Max E Valentinuzzi, EE, PhD, Emeritus Professor, Universidad Nacional de Tucumán, Argentina; Emeritus Investigator of Consejo Nacional de Investigaciones Cientificas y Técnicas (CONICET), Argentina

\author{
Abbreviations \\ ECG: Electrocardiogram; DC: Direct Current; AC: Alternat- \\ ing Current; VF: Ventricular Fibrillation
}

\section{Introduction}

Atrial fibrillation (AF), not to be confused with atrial flutter, is the term used to describe an irregular or abnormal heart rate. While AF and atrial flutter are similar, AF has more serious health implications such as an increased risk of having a stroke or a blood clot (thrombosis). The resting heart rate of someone without AF is usually between 60 and 100 beats per minute but this number is usually over 100 in AF. It is usually the result of an underlying condition such as hypertension (high blood pressure) or having an overactive thyroid but may develop for no known reason. In this circumstance, the person is said to have lone atrial fibrillation. There is some debate about the definition of lone AF. The term has been used to describe different characteristics. Some think it should not be distinguished as a type of AF at all. According to the American College of Cardiology and the American Heart Association, the term is currently used for people with AF who have no history or echocardiographic evidence of cardiovascular or pulmonary disease. Also included are those who do not have heart disease or are 60 years of age or younger and do not have any related conditions (heart failure, chronic obstructive pulmonary disease (COPD), diabetes, hyperthyroidism, acute infections, recent cardiothoracic or abdominal surgery, and systemic in- flammatory disease. AF can affect people at any age but is rare in children and is more common in the elderly population; it reaches about $0.5 \%$ of the world's population. According to the Centers for Disease Control and Prevention (CDC), of Atlanta, Georgia, USA, approximately $2 \%$ of people younger than 65 -yearsold have $A F$, while about $9 \%$ ages 65 and older have it (data as for Nov 26, 2018).

The main objective of the present article is to historically review the development and evolution of this important cardiac arrhythmia along with delving into its future possibilities.

\section{Definition}

Electrical cardioversion is a process by which the heart is shocked to convert it from an irregular rhythm back into a normal sinus rhythm. The ECG illustration above shows what the heart rhythm looks like before and after cardioversion. For patients in persistent atrial fibrillation, electrical cardioversion may be applied early in the process. For other AF patients, electrical cardioversion may not be tried until later, when medication has stopped working. While electrical cardioversion may be effective at converting the heart back into normal sinus rhythm, it may require multiple tries. The word version means to turn one organ from one condition into another [1]. Such concept is similar, say, to a given language version of a book first published elsewhere in another language.

\section{Origins}

A paper authored by two Russians researchers, 
Vishnevskil and Tsukerman, described electroshock therapy in some heart rhythm disorders. That was in 1966 [2]. Many years after, in 2009, cardioversion was updated by Cakulev, Efimov and Waldo [3]. Actually, Vishnevsky and Tsukerman performed the first reported cardioversion of AF using a DC shock in February 1959. The patient had had AF for 3 years and the restoration of normal sinus rhythm took place during mitral valve surgery. The same team reported the first successful transthoracic cardioversion of atrial arrhythmias in 20 patients using DC cardioversion in 1960. In 1970, Gurvich introduced the first biphasic transthoracic defibrillator, which became standard in Soviet medical practice from that time, preceding Western analogs by at least 2 decades [4].

\section{AF and Atrial Remodeling}

Three forms of atrial remodeling during a progression of atrial fibrillation (AF) have been described: Electrical, contractile and structural. Electrical remodeling is a consequence of high atrial rates and includes shortening of the refractory period of atrial myocytes and slowing of atrial conduction velocity. Structural remodeling is characterized both by changes in atrial myocytes in the interstitium and by changes in extracellular matrix composition and deposition of fibrotic tissue. Changes at the level of atrial myocytes include the loss of contractile structures and expression of fetal-like proteins, and accumulation of glycogen in the atrial interstitium.

To avoid having blood clots break free during the electrical discharge, it is of paramount importance that the patient takes an anticoagulant for one to two months before the procedure. Most likely, the patient is recommended not to have anything by mouth after midnight the night before. On arrival to the operating room, the patient will be fitted with an IV for receiving medications and fluids and connected to monitors. Once the patient is anesthetized, a defibrillator/cardioverter/pacemaker equipment will deliver a heart jolt of energy through paddles or ECG-type patches placed, say, on the front and back of the chest. This electric shock should restore the normal heart rhythm, and may take several tries. If external cardioversion fails, then internal cardioversion may be done and involves delivering the discharge through catheters inside the heart. Once the patient wakes up, he/she can go home, but will need someone to drive him/her. For a few days following the procedure, the chest is tender or the skin may have red patches. After the electrical cardioversion, antiarrhythmic drugs, and/or rhythm control medication may be administered. Besides, the anticoagulant medication must continue according to medical advice.

\section{Risks and Success Rates for Cardioversion}

Electrical cardioversion risks include skin burns, fluid in the lungs, heart attack, stroke, or even death, though that is very rare. Various studies have reported that cardioversion is over 90 percent effective in converting to a normal sinus rhythm though some people revert back into AF shortly thereafter. Success has been shown to be enhanced when patients are on an antiarrhythmic drug beforehand, which helps prevent reversion. Success depends on the size of the left atrium as well as how long the patient has been in AF. Patients with a very large left atrium, greater than 5 $\mathrm{cm}$, or who have been in constant AF for a year or two, may find that electrical cardioversion is not effective. Following a successful electrical cardioversion, the goal is to maintain a normal sinus rhythm, which only happens with about 20-30 percent of patients within the first year if they are not on antiarrhythmic drugs. Overall, the likelihood of reversion into atrial fibrillation is quite high, regardless of whether the patient is on rhythm control drugs. Several patients have mentioned that their electrical cardioversions were not successful in converting them to a normal sinus rhythm or in maintaining it. We know that AF patients with untreated sleep apnea are more likely to revert back than AF patients without sleep apnea. We recall a patient, close relative of one of us, who in the early 1960's lived for about 20 years with the arrhythmia. Cardioversion was then unknown in Argentina.

\section{Interesting Sad Learning Experience}

The behavior of the human being is often deceptive. As part of a trip to further international cooperation in medical research, in 1958, the well-known and influential senator Hubert $\mathrm{H}$. Humphrey [5] visited Moscow. Humphrey saw the Research Laboratory of General Reanimatology (Resuscitation), where he met its director, Dr. Vladimir Negovsky [6], and the laboratory's leading defibrillation researcher, Dr. Naum Gurvich (1905-1981) [7]. There, Humphrey saw experiments on the reversibility of death in animals through massive electric shocks. On return to the USA, he reported publically on such experiments and urged the development of programs through the National Institutes of Health on the physiology of death and resuscitation. Nevertheless, the work behind the Iron Curtain remained virtually unrecognized in the West.

\section{Work in the Western World After 1950}

In 1956, Paul Zoll [8] of Beth Israel Hospital and Harvard Medical School in Boston, MA, demonstrated successful closed-chest defibrillation in humans, again using an AC shock. Not long after, in 1960, working at Lariboisiere Hospital in Paris, France, an electrical engineer and physician, Fred Zacouto [9], completed the design of the first external automatic defibrillator/pacer. He had invented it in March 1953 and filed the related patent in July 1953 in Paris [10]. 


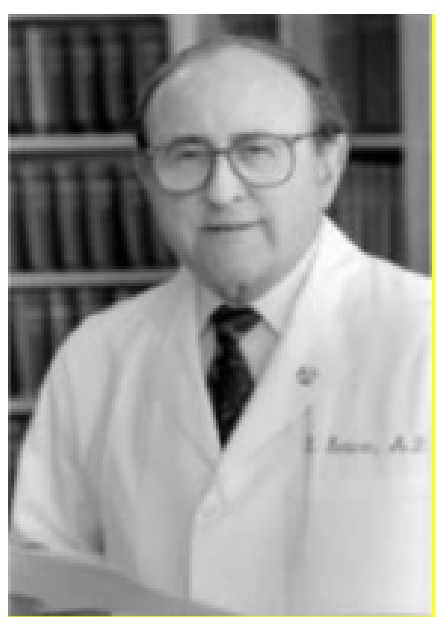

Figure 1: Bernard Lown, MD. Courtesy of Lown Cardiovascular Research Foundation.

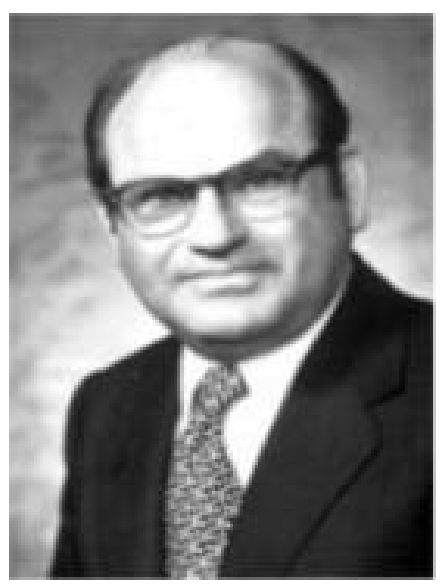

Figure 2: Baruch V Berkovits. Courtesy of the Heart Rhythm Foundation.

His Bloc Réanimateur was able to sense a slow pulse from an infrared device attached to different parts of patient's body (ear lobe and a finger) and provide transcutaneous pacing until spontaneous return of heart activity. At the same time, it could detect VF from an ECG and deliver an AC shock of adjustable voltage and duration with the ability to redetect VF and redeliver a shock if needed. It was first used to successfully defibrillate a patient in November 1960. A total of 68 devices were produced and sold by 1968, first by Zacouto's Savita company (mentioned in reference [3], but no information was found about such company) and later by Thomson-CFTH [11]. The device was used in hospitals in France, Switzerland, and Germany.

Bernard Lown (Figure 1), of the Peter Bent Brigham Hospital in Boston, MA, is credited in the Western world with initiating the modern era of cardioversion. He was the first in the West to combine defibrillation and cardioversion with portability and safety. In 1959 , in a patient with recurrent bouts of ventricular tachycardia (VT), Lown was the first to transthoracically apply AC shock using the Zoll defibrillator to successfully terminate an arrhythmia other than VF. This event is notable because intravenous administration of procainamide had failed to terminate the patient's VT, and application of the transthoracic shock became a dire necessity to try to save a human life. Because the procedure was unplanned and on an urgent basis and because there was not any information of which Lown's team was aware to provide data on the safety and efficacy of the procedure, it was done despite the hospital's resistance and only after Lown took sole responsibility. At that time, Lown knew little about defibrillation and the intricacies of AC versus DC shock. In early 1961, Lown, fortunately and quite incidentally, met a brilliant young medical engineer, Baruch Berkowitz (1926 - 2012) [12]. He created one of the variants of the defibrillator and an artificial pacemaker (Figure 2).

Baruch had been developing a DC defibrillator while working for the American Optical Corporation $(A O C)$ as the Director of Cardiovascular Research. Although the $A O C$ manufactured an $A C$ defibrillator, Berkovits was very aware of its shortcomings because he was familiar with the previous work of Gurvich. Thus, aware that DC shock was safer and more effective, Berkovits had decided to build a DC defibrillator for possible commercial use. Berkovits asked Lown if he would be interested in testing his device. In April 1961, Lown formally asked Berkovits to study his DC defibrillator in canines and for possible clinical application. A series of intense experiments followed that involved testing the efficacy of multiple waveforms and evaluating the safety of DC shock in a very large number of canines. During these experiments, the Lown-Berkovits investigation group, aware of the importance of avoiding the vulnerable period, introduced for the first time the novel concept of synchronizing delivery of the shock with the QRS complex. During these studies, they also developed a monophasic waveform with high efficacy and safety. Lown is credited with coining the term cardioversion for a synchronized shock during an arrhythmia other than VF. Lown subsequently went on to expand DC cardioversion to successfully convert both atrial and ventricular arrhythmias using the monophasic DC shock. This success promptly resulted in the acceptance and worldwide spread of DC cardioversion. One result of the success of the DC cardioverter-defibrillator was the development of the modern cardiac care unit, where Lown again played an important role. In 1962, Berkovits patented the DC defibrillator for the American Optical Corporation. Using a miniature capacitor developed for the US National Aeronautical and Space Administration, Frank Pantridge [13] together with John Anderson [14], a biomedical engineer, developed a $3.2 \mathrm{~kg}$ portable defibrillator that became available in 1971. Pantridge's ideas became widely accepted in the USA. Subsequently, Anderson 


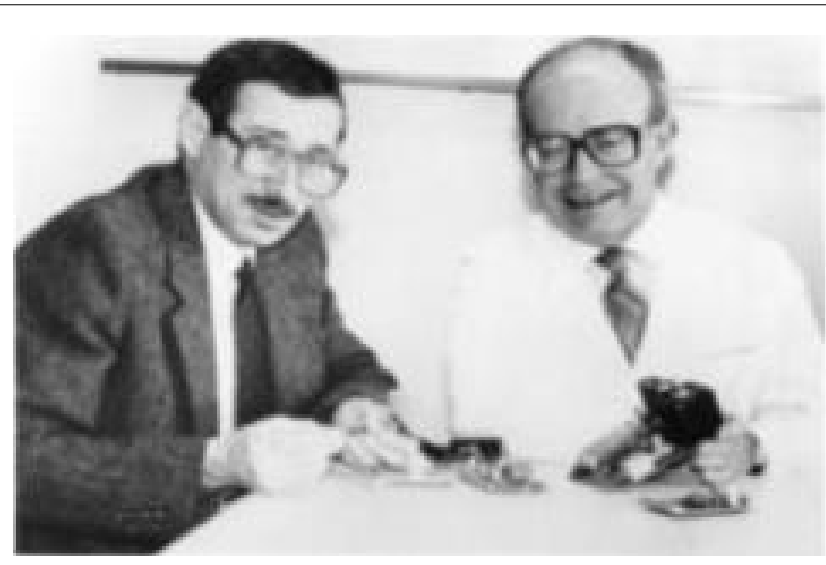

Figure 3: Drs. Morton Mower (left) and Michael Mirowski (right) with their first prototype of an automatic defibrillator. Courtesy of Ariella Rosengrand, MD.

and Jennifer Adgey [15], another physician from the Belfast group, were among the first to develop the semi-automatic and automatic portable external defibrillator in the late 1970's and early 1980's

\section{Mirowski and Mower}

Michael Mirowski (1924 - 1990), born in Poland, conceived the idea for an implantable cardiac defibrillator while in Israel, where he was trained at Tel Hashomer Hospital, Ramat Gan, Tel Aviv. In 1968, after a painful long period in different places, he could emigrate to the USA finding a place at Sinai Hospital, Baltimore, Maryland. He coined a sentence full of meaning: The bumps in the road are not bumps, they are the road.

Virtually simultaneously and independently, John Schuder, a PhD in Electrical Engineering and, thereafter, Associate Professor of Biophysics and Surgery at the University of Missouri, in Columbia, also began work on an implantable defibrillator [16]. In fact, Schuder was the first to implant and successfully use a cardiac defibrillator in a dog in January 1970 . He subsequently left the subject, instead concentrating on optimization of shocking waveforms. Schuder's continued contributions laid the foundation for the miniature, low-energy, reliable, high-voltage, biphasic waveform, which ultimately made contemporary Implantable Cardioverter Defibrillator (ICD) therapy possible. Mirowski eventually joined Cardiac Pacemakers Inc (CPI), where he met Mower [17] (Figure $3)$, persevered in his project overcoming many obstacles, from the enormous to the small. In February 1980, after 11 years of development, the first internal cardiac defibrillator was implanted in a patient at Johns Hopkins Hospital in Baltimore. After the third patient implantation, the device also included cardioversion. The cardioversion-defibrillation device obtained Food and Drug Administration approval in 1985.

\section{Discussion}

Little has changed the technique of cardioversion since Lown's article in the early 1960s. Progress has been made in reducing the already low associated complication rate and in understanding the factors responsible for success. Successful cardioversion or defibrillation occurs when a shock with sufficient current density reaches the myocardium. Because the maximum energy stored in the capacitor is fixed, the principal determinant of current density is transthoracic impedance to $D C$ discharge. The factors influencing trans-thoracic impedance that can be modified by the technique of cardioversion include the interface between the electrode and skin, the electrode size, and the electrode placement. Although a variety of chest placements have been used, there are two conventional positions for the electrode paddles: Anteroposterior and anterolateral. In the anterolateral position, paddles are placed between the ventricular apex and the right infraclavicular area, whereas in the anteroposterior position, one paddle is placed over the sternum and the second interscapularly. Lown originally advocated that the anteroposterior position is superior because it requires less energy to reverse AF. Some studies have confirmed this notion, whereas others have shown no advantage to either paddle position. Because only $\approx 4 \%$ to $5 \%$ of the shocking energy actually reaches the heart, minor deviation of this electric field probably has little effect on the final outcome. In today's era of biphasic waveforms, the position of the paddles most likely plays an even smaller role.

The size of the electrodes through which the shock is delivered has been shown to significantly influence the transthoracic impedance. A larger electrode leads to lower impedance and higher current, but an increase in size of the electrode beyond the optimal size leads to a decrease in current density. In humans, paddle electrode size with a diameter between 8 and $12 \mathrm{~cm}$ appears to be optimal. Improved skin-to-electrode contact also leads to reduction of transthoracic impedance and an increase in the success rate. Hand-held paddles may be more effective than self-adhesive patch electrodes, perhaps because of better skin contact. In addition, the usage of non-salt-containing gels has been associated with an increase in transthoracic resistance.

The biphasic waveform deserves a special comment. Gurvich was the first to demonstrate its superiority over the monophasic in dogs in 1967. Most of the external defibrillators in the Soviet Union from the early 1970s used it, and are known in Russia as the Gurvich-Venin waveform [18]. It took much longer for the West to realize its benefit over the original Lown monophasic waveform. The first experiments comparing the monophasic and biphasic waveforms for transthoracic defibrillation were done independently by Schuder, et al. in the 1980s. The efficacy of an ICD is limited by the maximum stored energy. In their attempt to limit the device size, manufacturers of the 
ICD finally chose the more effective biphasic waveform. Although the Gurvich-Venin biphasic waveform was superior to the monophasic waveform, its requirement for an inductor precluded major reduction in size for use in ICDs. It was the work of John Schuder and also Raymond Ideker [19], then at Duke University, on optimization of biphasic waveforms that made miniaturization of implantable defibrillators possible. After 2000, most defibrillators developed for either external or internal use were "biphasic" devices, meaning that they reverse polarity 5 to 10 $\mathrm{ms}$ after the discharge begins. The biphasic waveform has been shown in humans to defibrillate both $A F$ and VF more effectively than monophasic waveform. The recommendation says to start with $200 \mathrm{~J}$ biphasic waveforms, particularly when cardioverting patients with AF of long duration. Successful termination of organized tachycardias requires less energy than disorganized rhythms such as polymorphic VT, AF, or VF. Similarly, tachycardias of shorter duration have higher immediate conversion success rates. For instance, the overall success rate in restoring sinus rhythm in patients with $A F$ is $\geq 90 \%$ when the arrhythmia is of $<1$ year's duration compared with $50 \%$ when AF has been present for $>5$ years. The risks associated with DC cardioversion are related mainly to inadvertent initiation of new tachyarrhythmias, the unmasking of bradycardia, and postshock thromboembolism. More than $25 \%$ of patients have bradycardia immediately after cardioversion, and this incidence is higher in patients with underlying sinus node dysfunction. Ventricular arrhythmias are uncommon after cardioversion unless an unsynchronized shock was applied, VT previously existed, or digitalis toxicity was present. In the latter instance, DC cardioversion is contraindicated. A major risk associated with cardioversion is thromboembolism. Thromboembolic events are more likely to occur in patients with AF who have not been anticoagulated adequately before cardioversion. The incidence varies and has been reported to be between $1 \%$ and $7 \%$. In a large series, the incidence was reduced to $0.8 \%$ from $5.3 \%$ with proper anticoagulation. Nevertheless, the efficacy and safety of cardioversion in its current form have withstood the test of time, and it continues to be used widely by clinicians as the most frequent approach to restoring sinus rhythm. This success, associated with a very favorable risk profile, has initiated a trend toward wider use of cardioversion/defibrillation not only by medical personnel but also by the general public. Although portable automatic external defibrillators have existed since 1979, the accumulation of clinical studies confirming their safety, efficacy, and diagnostic accuracy has recently prompted several US federal initiatives to expand public access to defibrillators.

It is hard to imagine the changes that the future may bring to a technique that has changed so little over the last several decades. Currently and by far, the vast majority of sudden cardiac death episodes occur in subjects without any identifiable or recognized heart disease. Current attention is focused only on the relatively small percentage of patients with identifiable or recognized risk factors for sudden cardiac death, mainly subjects with structural heart disease. Eventually, the use of defibrillation may be similar to the current use of seat belts. If the risks are sufficiently low and major inconveniences are avoided, there would be a good reason to expand their use to populations at much lower relative risk for sudden cardiac death.

Another very important area for future improvement would be to further reduce the defibrillation threshold. This would serve the ultimate goal of eventually eliminating pain, anesthesia, and sedation during shocks, if possible. To achieve this, several different strategies, perhaps in combination with each other, will be used. Current research points toward the direction this is already taking. In all likelihood, more effective cardioversion/defibrillation waveforms will be used. Shocks from $\geq 2$ sites simultaneously or sequentially will further improve cardioversion/defibrillation effectiveness. In addition, combination of shocks with cardiac pacing may prove particularly useful. It is known that pacing can influence and terminate reentrant or triggered arrhythmias. Work on animal models and humans on the mechanisms of VF and AF suggests the presence of one or more drivers that may make the strategy of combining shocks with pacing plausible. The hope would be that this combination will result in the need for less energy to restore normal sinus rhythm. This would certainly benefit internal as well as external cardioversion/defibrillation. Clearly, this approach requires more work on the mechanisms of these arrhythmias and the technology used to cardiovert and defibrillate them.

It is surprising to learn how much seminal work had been done behind the Iron Curtain that was almost completely unknown in the West. For too many years, humanity was deprived of a life-saving treatment. It was fortunate that Barouh Berkovits bridged the gap between East and West by making the DC transthoracic cardioverter/defibrillator available to Dr. Bernard Lown.

\section{References}

1. Valentinuzzi Max E (2011) Cardiac Fibrillation-Defibrillation: Clinical and Engineering Aspects. Series on Bioengineering and Biomedical Engineering, volume 6, World Scientific Publisher (WSP), Singapore, 279.

2. Vishnevskiĭ AA, Tsukerman BM (1966) Electroshock therapy of disorders of heart rhythm. Eksp Khir Anesteziol 11: 39-53.

3. Ivan Cakulev, Igor R Efimov, Albert L Waldo (2009) Cardio- 
version: Past, Present, and Future. Circulation 120: 16231632.

4. Igor R Efimov (2009) Naum Lazarevich Gurvich (19051981) and his contribution to the history of defibrillation. Cardiol J 16: 190-193.

5. Hubert Horatio Humphrey Jr (1911-1978) American politician who served as the 38th Vice-president of the United States from 1965 to 1969.

6. Peter Safar (2001) Vladimir A Negovsky: The father of reanimatology. Resuscitation 49: 223-229.

7. Ludmila $\vee$ Ussenko, Alexander V Tsarev, Yaroslav A Leschenko (2006) Naum L Gurvich: A pioneer of defibrillation. Resuscitation 70: 170-172.

8. Paul Maurice Zoll (1911-1999) Jewish American cardiologist, one of the pioneers of the artificial cardiac pacemaker and cardiac defibrillator.

9. Fred Zacouto, Louis Guize, Pierre Maurice, Alain Gerbaux (1973) Orthorhythmic pacing in arrhythmias. American J Cardiology 31: 165.

10. Fred Zacouto (1977) Method of stimulating the heart. Patent number 4052991.

11. In 1893 Compagnie Française Thomson-Houston (CFTH) was set up as a partner to General Electric (GE). It is from this company that the modern Thomson companies would evolve.

12. Bercovits, born in Czechoslovakia. Parents and sister died in Auschwitz. In the 1950s he emigrated to the USA. From 1975 until his retirement he worked for Medtronic, Minnesota. In 1982, awarded by the Heart Rhythm Community.

13. James Francis "Frank" Pantridge (1916-2004), physician and cardiologist, from Northern Ireland, who transformed emergency medicine and paramedic services with the invention of the portable defibrillator.

14. John Anderson (1942-2012) was co-founder, director, and the chief technology officer of Intelesens Ltd. and HeartSine $^{\circledR}$ Technologies Inc. Anderson formed the Northern Ireland Bio-Engineering Centre (NIBEC) and was its first director.

15. Jennifer Adgey, well-known and highly respected cardiologist, at Royal Victoria Hospital, Belfast, United Kingdom of Great Britain \& Northern Ireland, specialized in Acute Coronary Syndromes, Acute Cardiac Care, Ventricular Arrhythmias and Sudden Cardiac Death.

16. John Claude Schuder, born 1922, Illinois. Schuder was Instructor in Electrical Engineering at Purdue University from 1949 to 1954, and Associate Professor 1954-1956. He was Fellow of Surgical Research 1957-1959 and Associate Professor of Electrical Engineering in Surgical Research from 1959-1960 at the Pennsylvania Medical School. In 1960 Schuder joined the University Medical School in Columbia as Associate Professor of Biophysics in Surgery, promoted to Professor in 1964.

17. Mower, born in Baltimore. 1955, Johns Hopkins University graduate. 1959, Maryland Medical School graduate. He was cardiology fellow of Sinai Hospital. Later, Vice-President of CPI (Cardiac Pacemakers, Inc). In 1995 senior consultant of Guidant Corporation, company that followed the former. He retired in 1996.

18. Vyacheslav A Vostrikov, Boris B Gorbunov, Sergey V Selishchev (2015) Igor V. Venin Founder of biphasic waveform defibrillators. Journal Biomedical Science and Engineering 8: 345-349.

19. Raymond E Ideker (2019) HRS 40th anniversary viewpoints: What's it all about? Heart Rhythm 16: 484-485. 